\title{
Prediction Role of Working Memory and Sustained Attention in Sleep Quality of Children with Attention Deficit- Hyperactivity Disorder
}

\author{
Jahanbakhsh Bakhtiari, Sayed Abbas Haghayegh*
}

Department of Clinical Psychology, Najafabad Branch, Islamic Azad University, Najafabad, Iran

Article Info:

Received: 3 Jul 2019 Revised: 22 Sep 2019

Accepted: 4 Dec 2019

\section{ABSTRACT}

Introduction: The purpose of this study was to investigate the relationship between sleep problems, working memory, and sustained attention of children diagnosed with attention deficit hyperactivity disorder (ADHD). Materials and Methods: A correlation study has been used for this investigation. The statistical population of this study was all students who have been diagnosed with ADHD in the elementary school of the Department of Education in District 3 of Isfahan, who studied in the school year of 2017-2018. A questionnaire containing questions about Behavioral Rating Scale and Sleep Quality was provided to the parents of 200 students aged 7-11 years old. After removing unskilled questionnaires, based on the cut-off score, the Acne Scale of the Conner Questionnaire consisted the active memory of Beck and the Continuous Performance Test were performed in 65 students with ADHD. Results: The results have shown a negative and significant relationship between sleep problems and active memory of children with ADHD. However, there was no significant relationship between sleep problems and sustained attention. Conclusion: The results suggest that sleeping problems can reduce the performance of active memory and affect the cognitive functions of children.
Key words:
1. Attention Deficit Disorder with Hyperactivit
2. Sleep
3. Behavior Rating Scale

*Corresponding Author: Sayed Abbas Haghayegh

E-mail:abbas-haghayegh@yahoo.com 
نقـش ييشبينـى كنندهُ حافظهُ فعال و نكَهدارى توجه در كيفيت خواب كودكان واجد اختلال نقص تمد توجه -بيش فعالى

\section{جهانبخش بختيارى، سيد عباس حقايق"}

كروه روانشناسى، واحد نجف آباد، دانشعاه آزاد اسلامى، تجف آباد، ايران

\section{اطلاعات مقاله:}

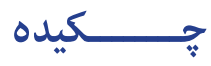

مقدمه: هدف از اين يروهش بررسى ارتباط بين مشكلات خواب، حافظهٔ فعال و نكَهدارى توجه كودكان

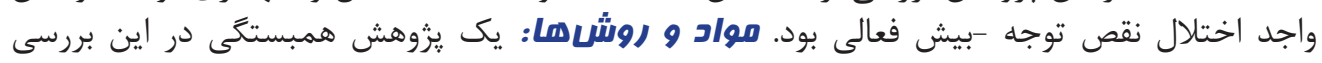

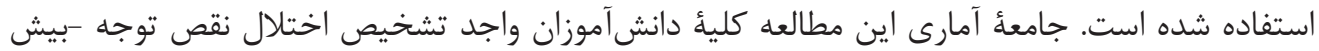

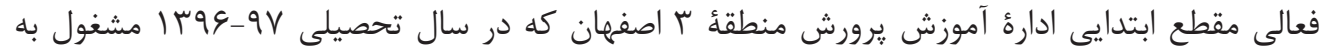

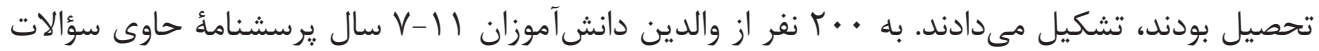

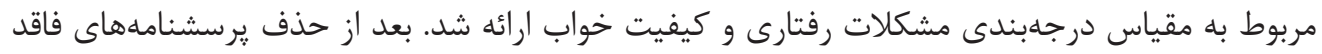

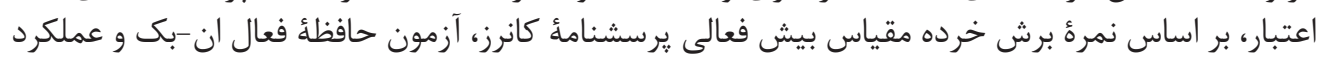

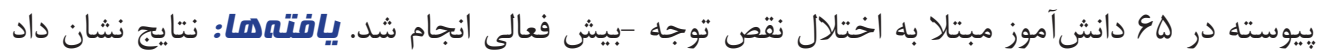

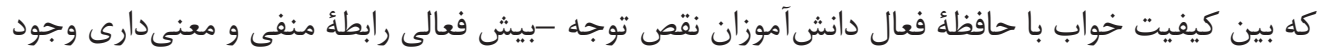

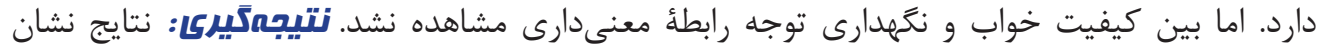

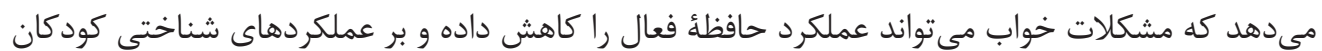
تأثير بخذارد. 
شـامل: يـك سيسـتم اجرايسى مركـزى عمومسى و دو سيسـتم

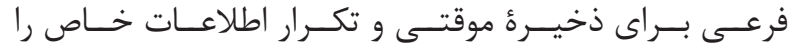

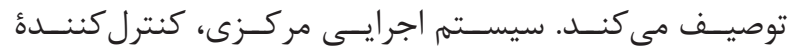

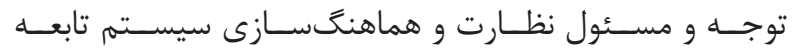

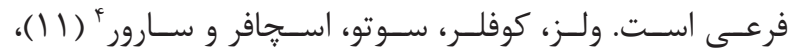

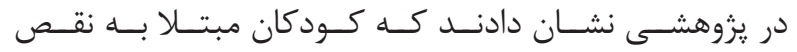

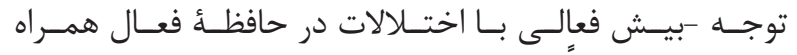

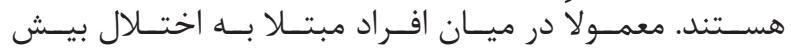

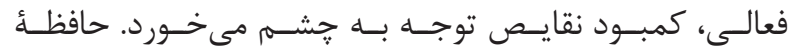

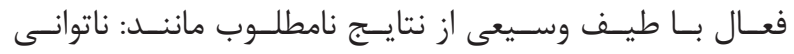

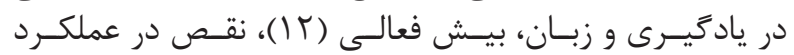

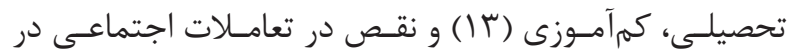

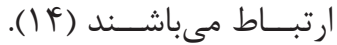

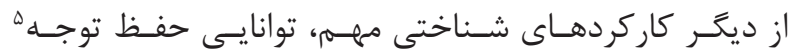

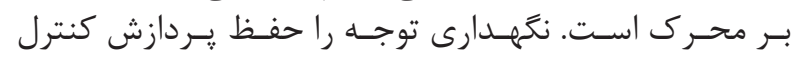

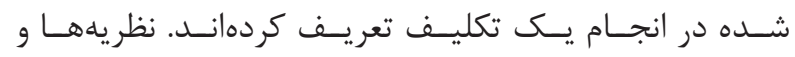

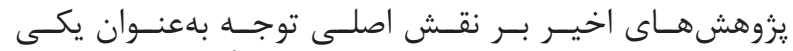

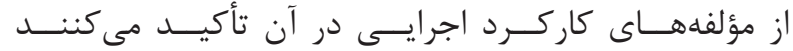

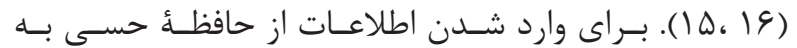

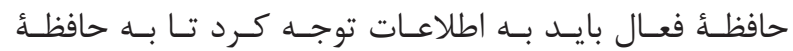

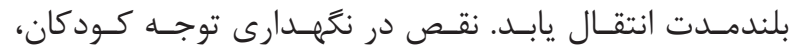

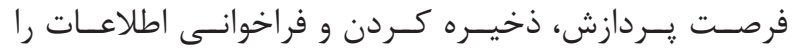

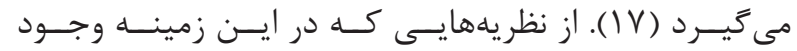

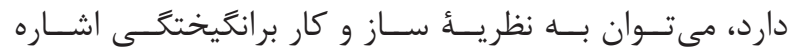

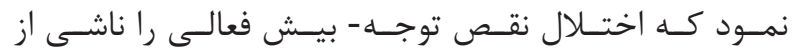

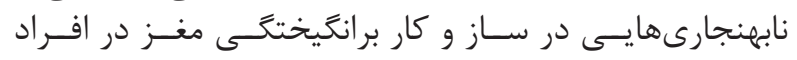

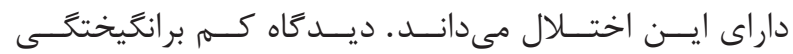

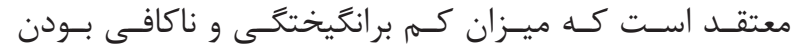

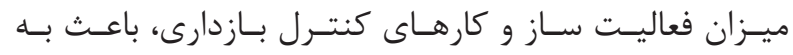

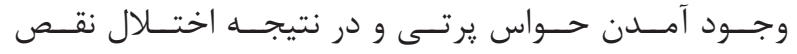

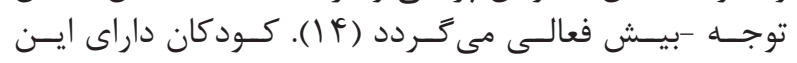

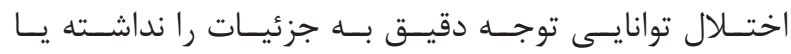

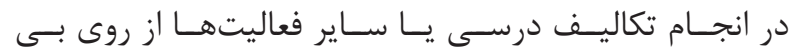

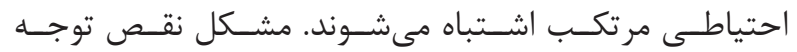

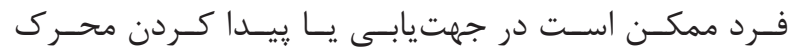

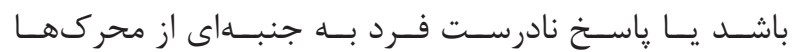

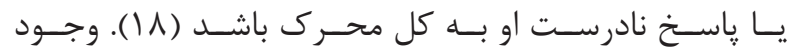

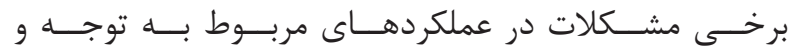

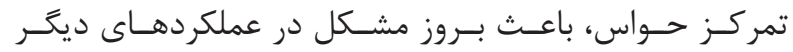

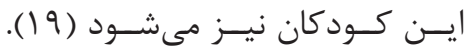

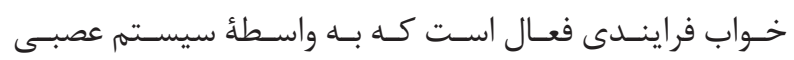

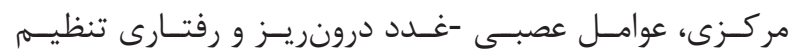

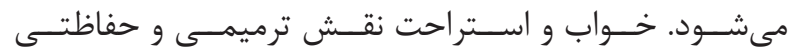

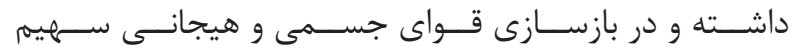

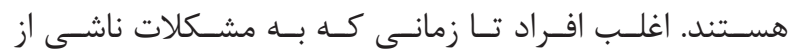

${ }^{1}$ Attention deficit hyperactivity disorder

${ }^{2}$ Working memory

${ }^{3}$ Baddeley

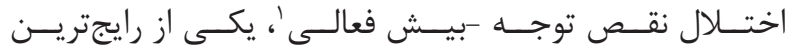

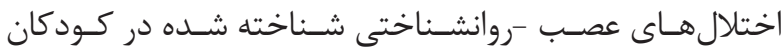

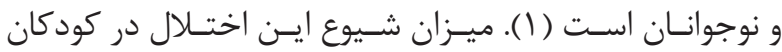

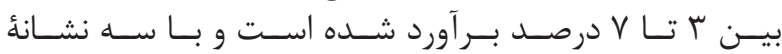

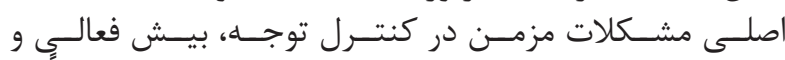

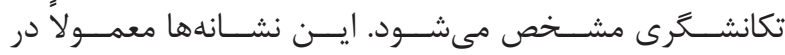

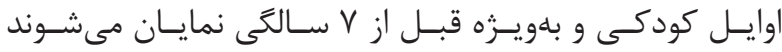

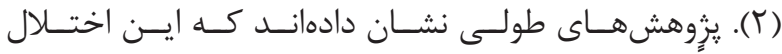

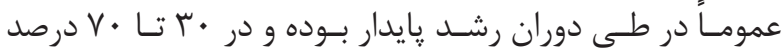

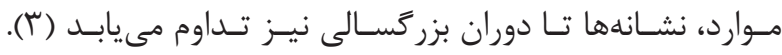

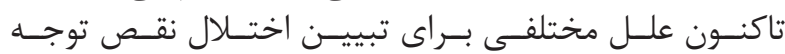

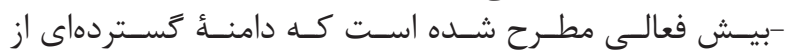

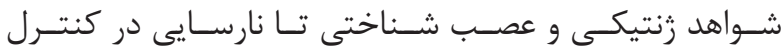

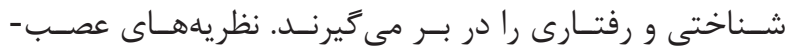

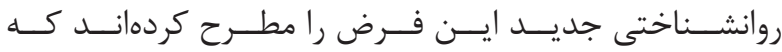

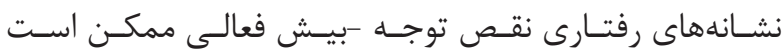

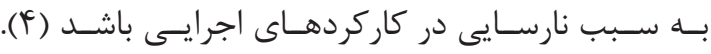

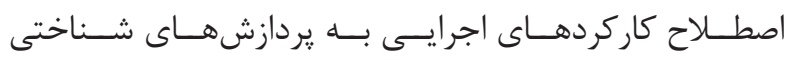

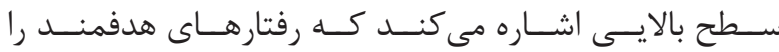

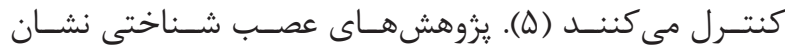

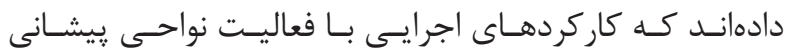

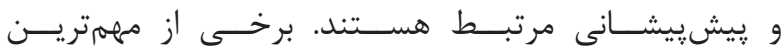

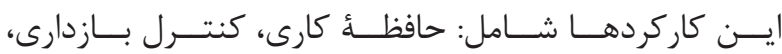

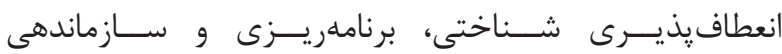

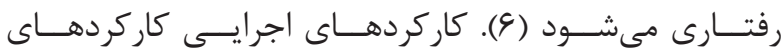

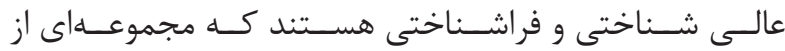

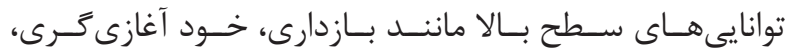

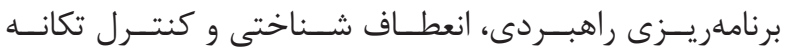

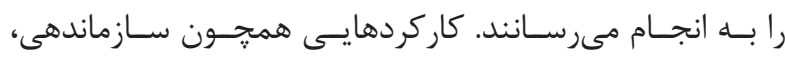

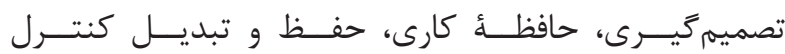

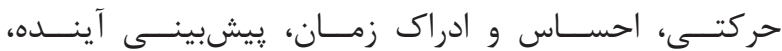

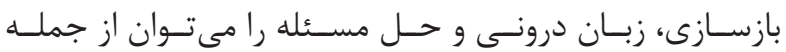

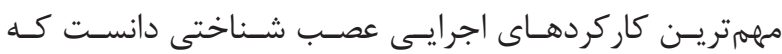

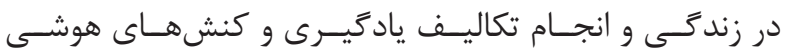

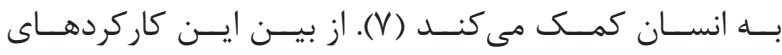

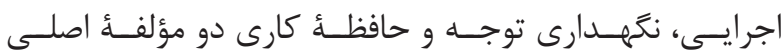

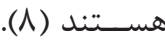

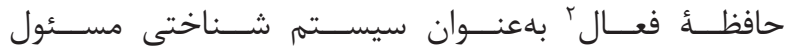

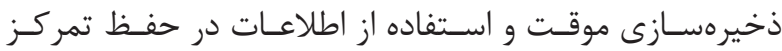

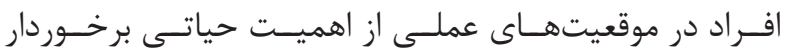

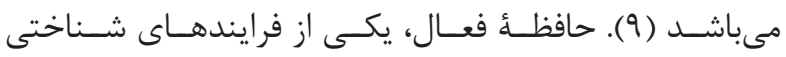

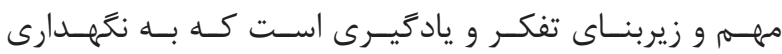

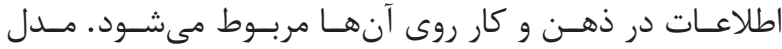

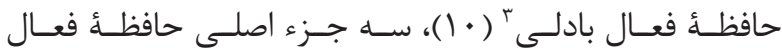

${ }^{4}$ Wells, Kofler, Soto, Schaefer and Sarverd ${ }^{5}$ Sustained attention 


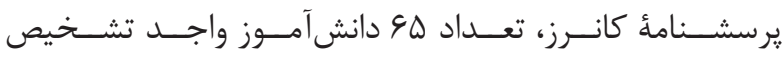

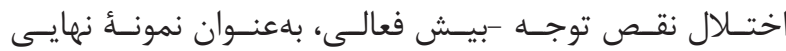

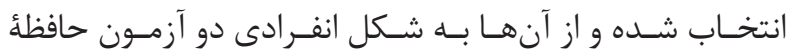

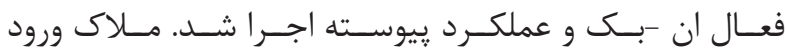

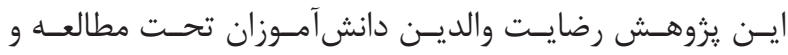

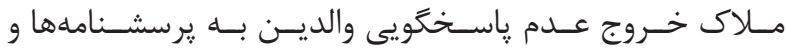

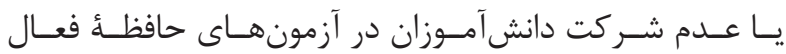

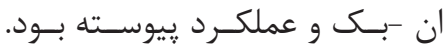

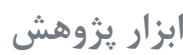

\section{مقياس درجهبندى كانرز (فرم والدين)}

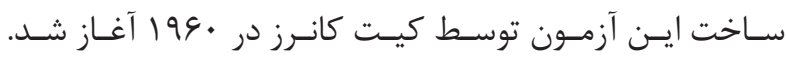

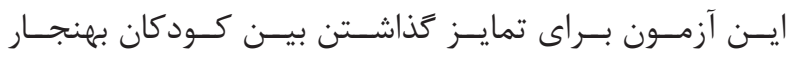

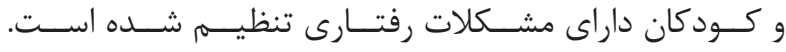

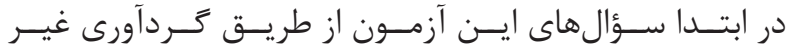

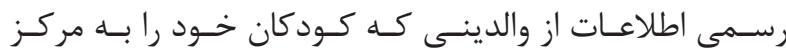

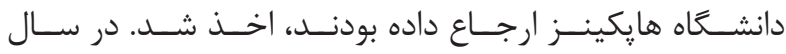

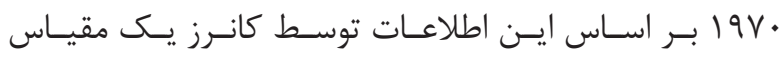

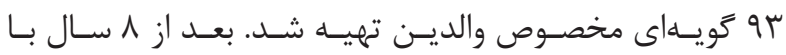

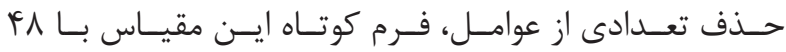

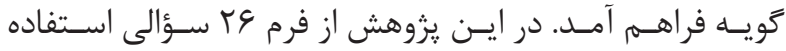

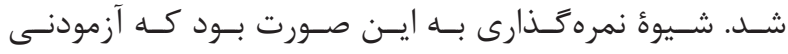

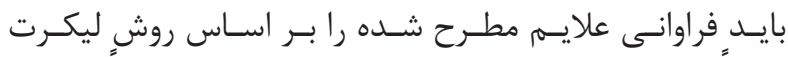

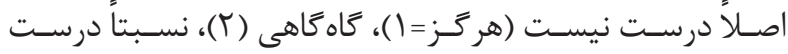

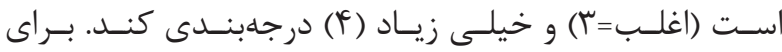

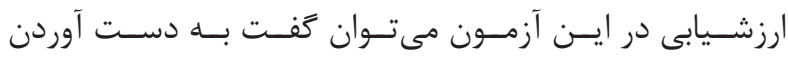

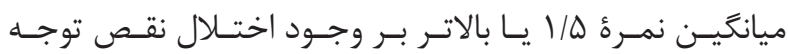

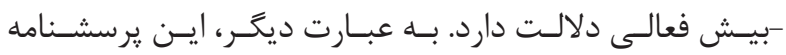

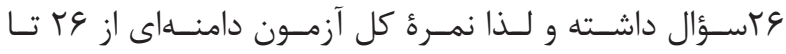

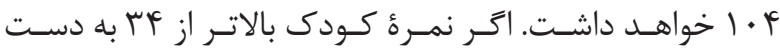

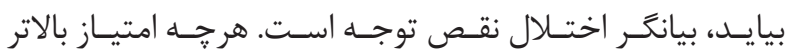

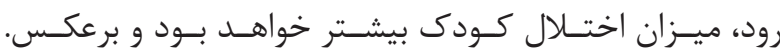

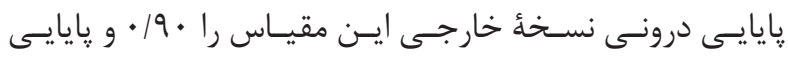

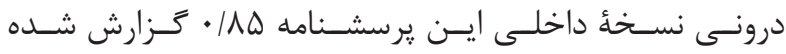

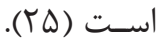

يرسشنامه خواب

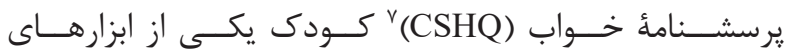

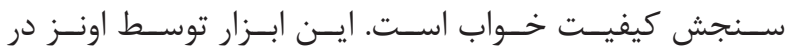

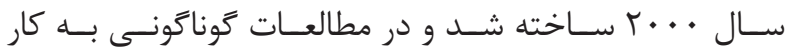

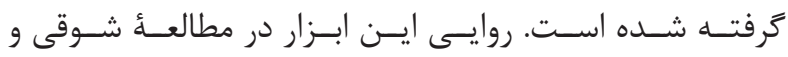

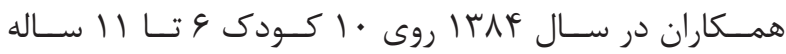

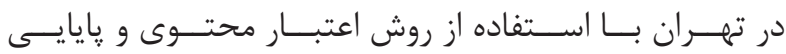

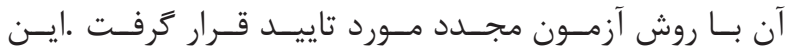

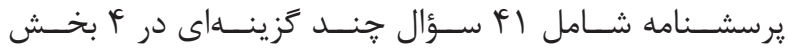

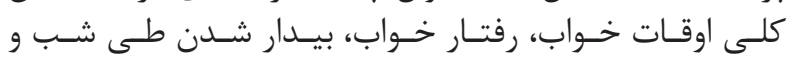

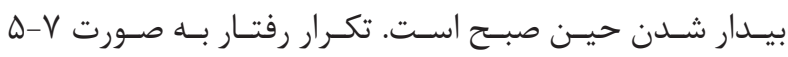

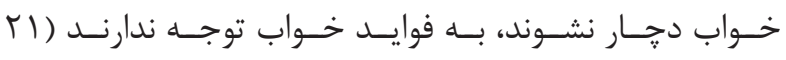

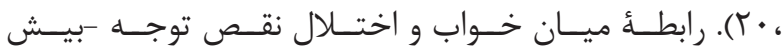

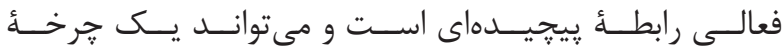

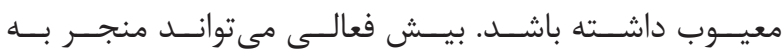

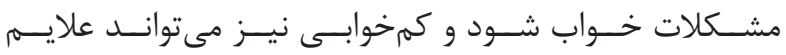

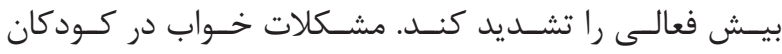

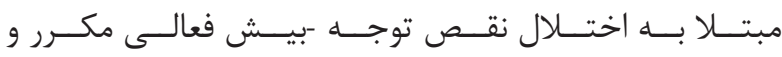

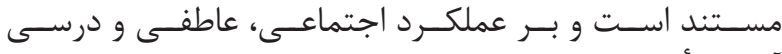

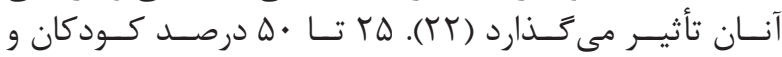

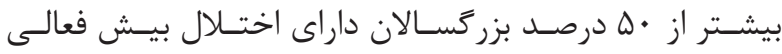

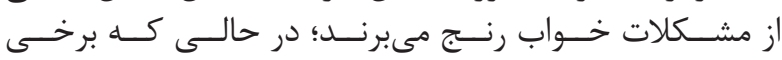

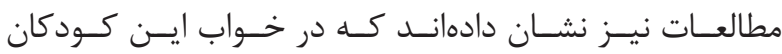

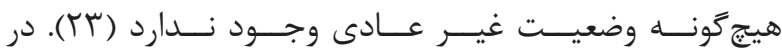

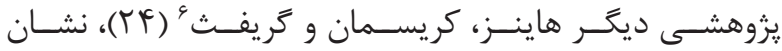

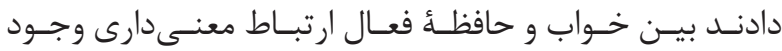

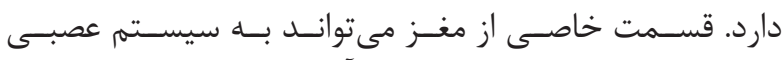

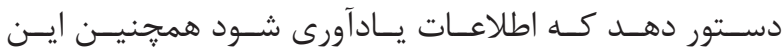

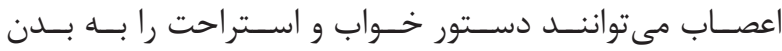

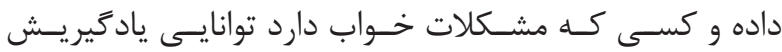

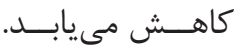

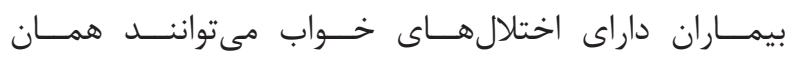

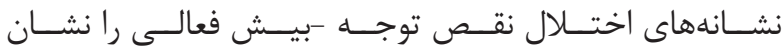

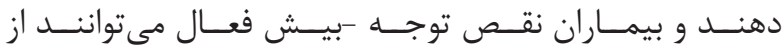

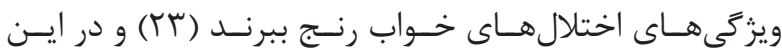

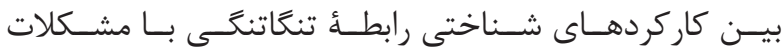

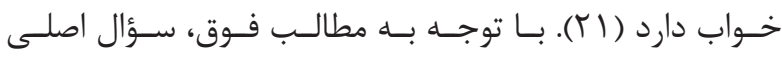

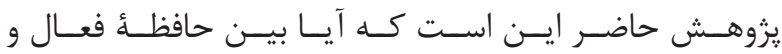

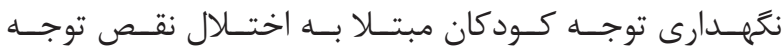

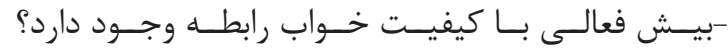

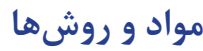

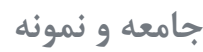

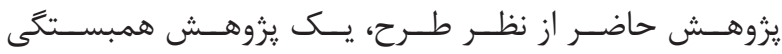

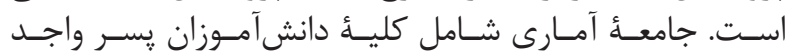

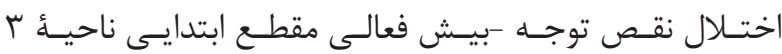

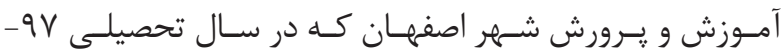

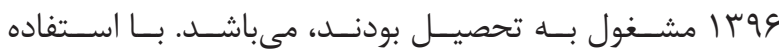

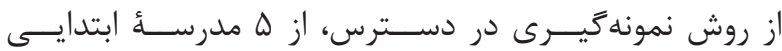

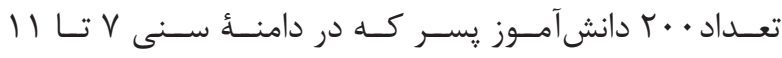

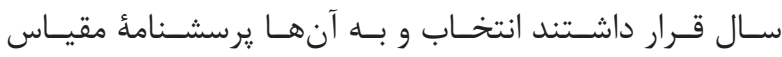

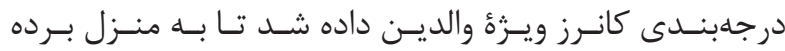

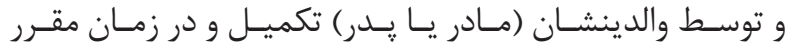

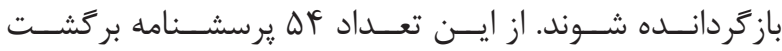

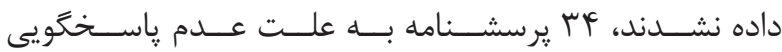

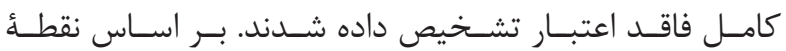

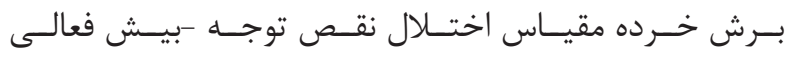




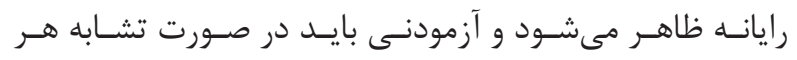

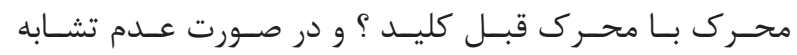

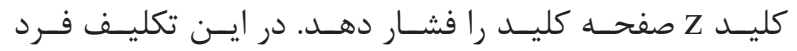

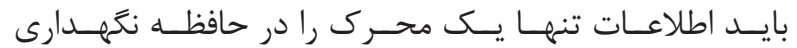

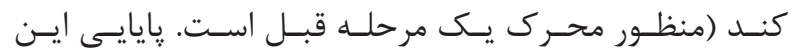

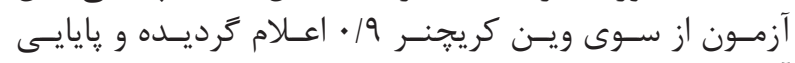

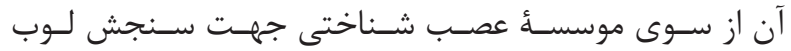

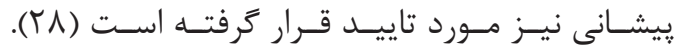

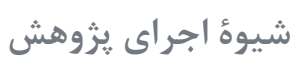

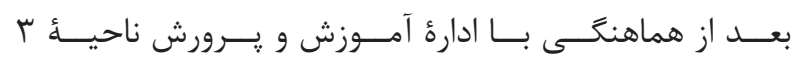

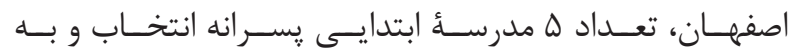

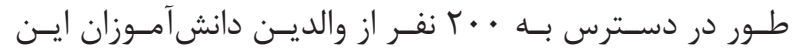

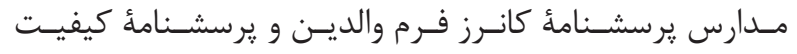

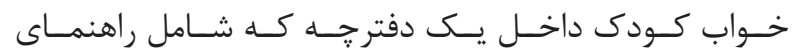

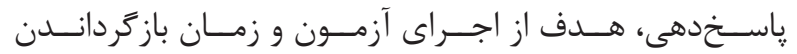

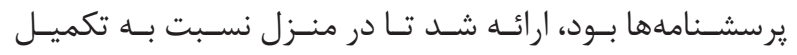

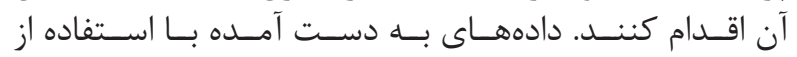

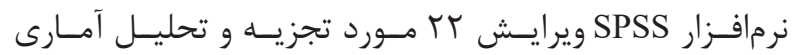
قـرار عرفت تجزيه و تحليل دادهها

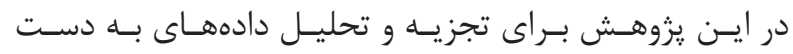

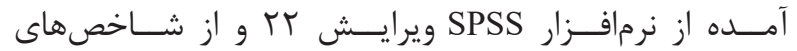

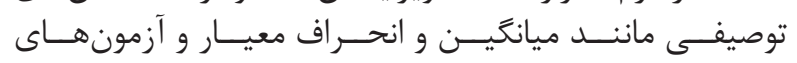

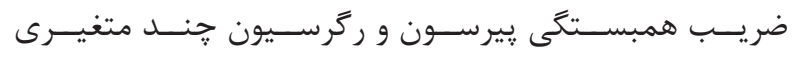

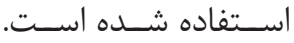

بافته بافا

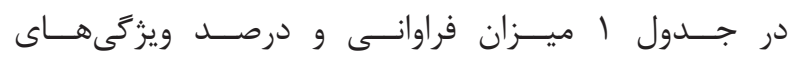

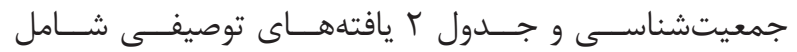

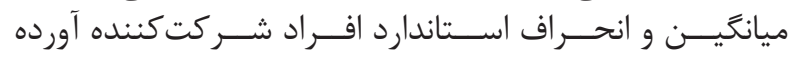
شــده اســـت انحت

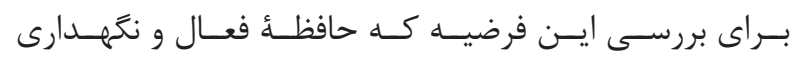

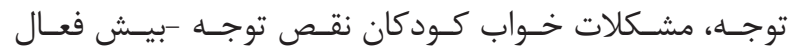

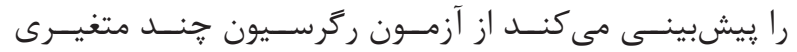

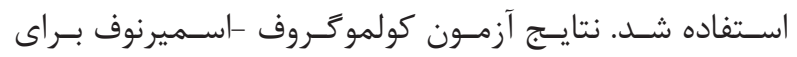

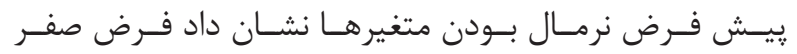

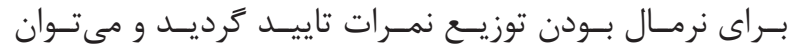

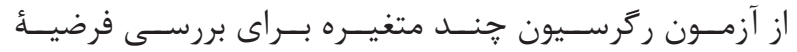

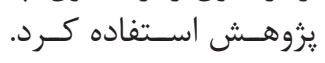

در جداول fا و ه نتايج ضريب ركرسيون آورده شده است.

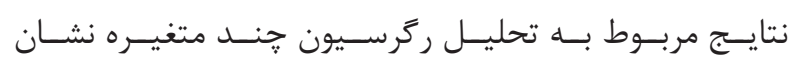

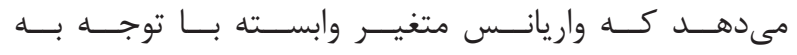

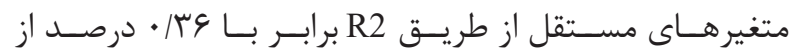

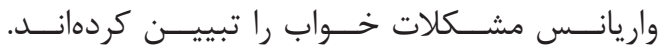

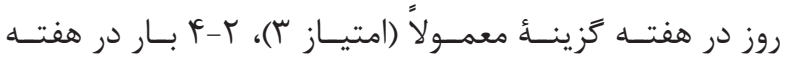

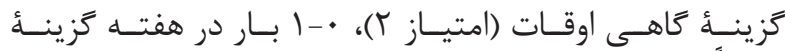

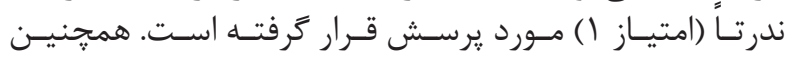

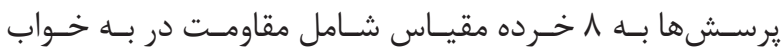

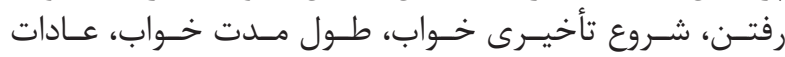

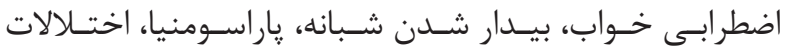

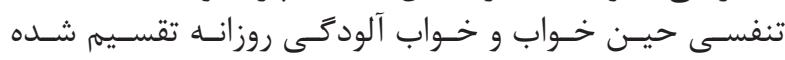

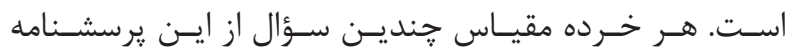

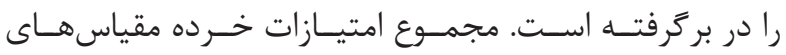

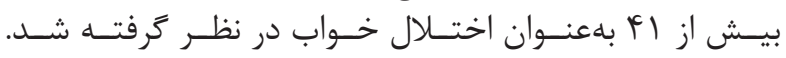

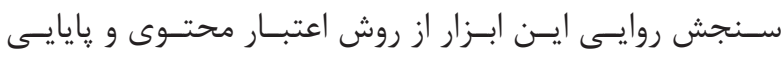

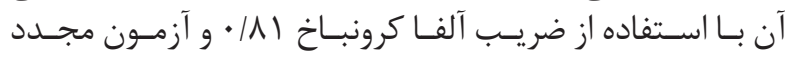

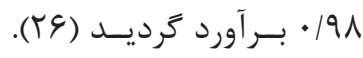

\section{آزمون نگهدارى توجه: عملكرد ييوسته}

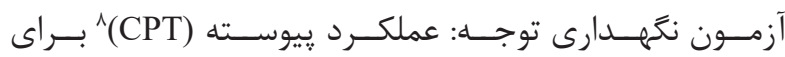

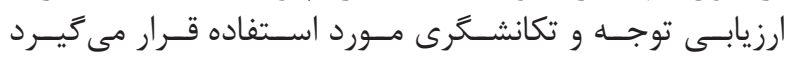

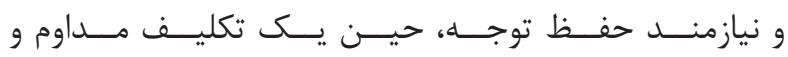

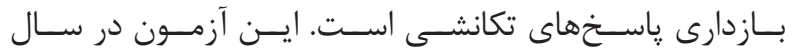

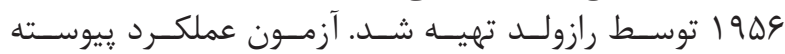

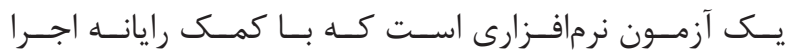

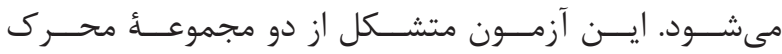

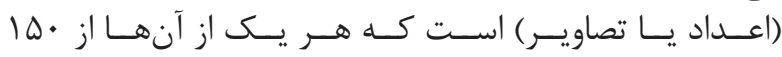

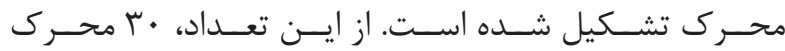

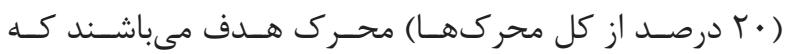

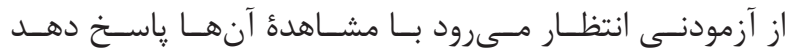

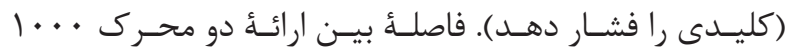

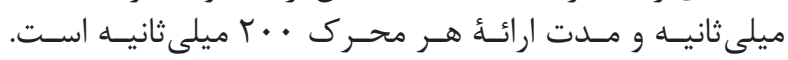

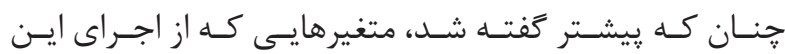

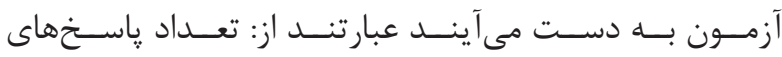

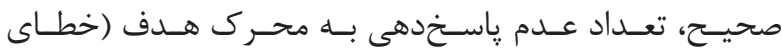

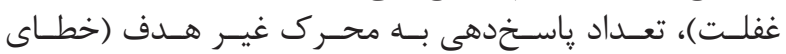

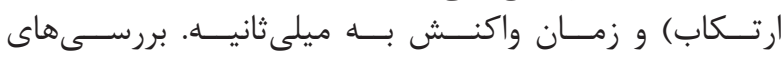

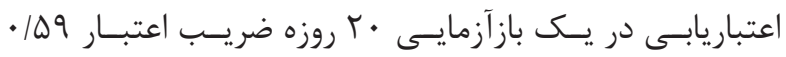

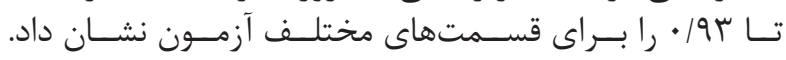

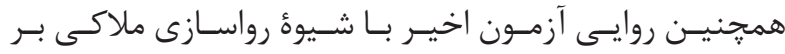

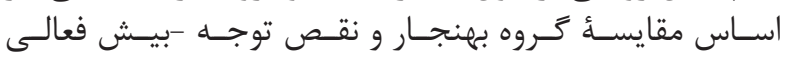

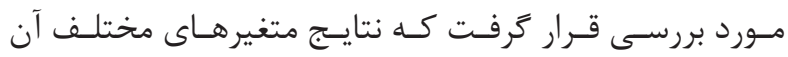

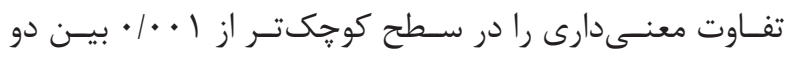
كَروه نشـان داد (TV)

\section{آزمون حافظة فعال N-Back}

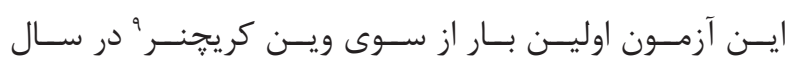

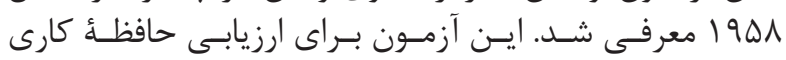

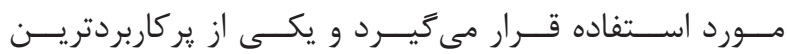

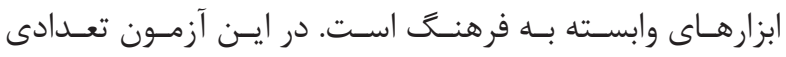

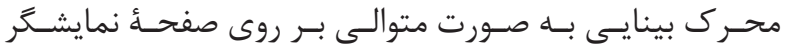

${ }^{8}$ Continuous performance test

${ }^{9}$ Kirchner 
جدول ا- فراوانى و درصد فراوانى ويرَّىهاى جمعيتشناسى افراد شركت كننده.

\begin{tabular}{|c|c|c|c|c|c|c|c|}
\hline درصد & فراواتى & سطوح & متغير & درصد & فراوانى & سطوح & متغير \\
\hline$\Gamma / 1$ & $r$ & زير دييلم & \multirow{4}{*}{ تحصيات مادر } & r & 10 & بدر & \multirow{2}{*}{ نسبت تكميل كنينده با فرد } \\
\hline$r V / V$ & 11 & دييلم & & $v 9 / 9$ & $\Delta$. & مادر & \\
\hline$\Delta \Delta / \Delta$ & re & ليسانس & & $\mathrm{V} / \mathrm{V}$ & $\Delta$ & - & \multirow{5}{*}{ تعداد خخواهر برادرها } \\
\hline I & 9 & بالاتر از ليسانس & & $\Delta \Delta / f^{\circ}$ & 48 & 1 & \\
\hline $19 / 9$ & 11 & زير ديبلم & \multirow{4}{*}{ تحصيلات بدر } & re/ & IV & r & \\
\hline FVIV & r) & ديِلم & & $9 / \pi$ & 9 & $r$ & \\
\hline rV/V & 11 & ليسانس & & $1 / \Delta$ & 1 & $f$ & \\
\hline$V / V$ & $\Delta$ & بالاتر از ليسانس & & IT/ & rT & اول & \multirow{5}{*}{ ترتيب تولد } \\
\hline$r q / r$ & 19 & كمتر از ه/ا ميليون & \multirow{4}{*}{ سطح اغتصادى } & $+\pi / 1$ & 广^ & 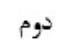 & \\
\hline $94 / 9$ & fr & بين ه/ آتا ميليون & & $\mid \Delta / f^{4}$ & 1. & سوم & \\
\hline g/r & f & بيشتر از ب ميليون & & $9 / 4$ & f & جهارم & \\
\hline $1 \ldots$ & 90 & كل & & $1 / \Delta$ & 1 & بنجم بن & \\
\hline
\end{tabular}

جدول r- آمار توصيفى متغيرهاى ثروهش.

\begin{tabular}{|c|c|c|c|c|c|}
\hline حداكثر & حداقل & انحراف استاندارد & ميانگين & تعداد & متغيرها \\
\hline r人 & 10 & $9 / 4 \wedge$ & r/r & 90 & كيفيت خواب \\
\hline $1 \Delta V$ & 114 & $11 / 19$ & ITF/TE & 90 & نغمدارى توجه \\
\hline rFA & $1 \cdot V$ & re/qA & 19.1A & 90 & حافظئ فعال كل \\
\hline
\end{tabular}

جدول r- آزمون كولموكروف -اسميرنوف براى بيش فرض نرمال بودن نمرات.

\begin{tabular}{|c|c|c|}
\hline سطح معنى دارى & مقدار z & متغير \\
\hline . $/ \Delta r$ & ·/ $/ 1$ & نَخهدارى توجه \\
\hline$\cdot / f I$ & $1 / V V$ & حافظةُ فعال \\
\hline - /人F & $1 / K \omega$ & مشكلات خواب \\
\hline
\end{tabular}

جدول F- Pتايج ركرسيون קند متغيره متغيرهاى پِيشبين با كيفيت خواب.

\begin{tabular}{|c|c|c|c|c|c|c|c|}
\hline R2 & $\mathrm{R}$ & سطح معنى دارى & $\mathrm{F}$ & ميانكين مجذورات & مجموع مجذورات & درجه آزادى & مدل \\
\hline . & .19. & $\cdot / \cdots$ & IV/9Y & fqr/दr & r & $Q \Lambda V / r \Delta$ & رَرسيون \\
\hline- & - & - & - & $r V / \Delta T$ & Gr & $1 \cdot V \cdot V / r \cdot$ & باقىمانده \\
\hline - & - & - & - & - & sf & $r \varepsilon q \uparrow / \Delta Q$ & كل - كل \\
\hline
\end{tabular}

جدول ه- نتايج حاصله از ركرسيون خند متغيرؤ فرضيات تحقيق.

\begin{tabular}{|c|c|c|c|c|c|}
\hline سطح معنى دارى & مقدار t & Beta & خطاى استاندارد & B & متغيرها \\
\hline • $\mid \Delta T$ & $\cdot 194$ & $\cdot 1 \cdot 09$ & $\cdot 1 \cdot 09$ & $\cdot / \cdot r V$ & نخهرارى توجه \\
\hline$\cdot 1 \cdot \cdots$ & $-\Delta / \Lambda \Lambda$ & -.1098 & $.1 \cdot 19$ & $-\cdot / 11$ & حافظهُ فعال \\
\hline
\end{tabular}

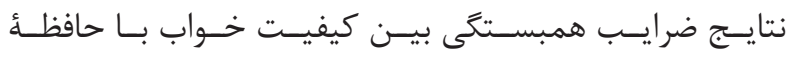

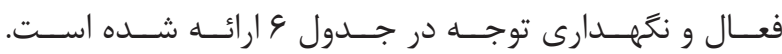

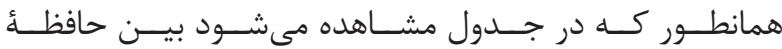

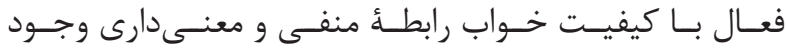

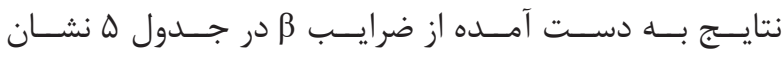

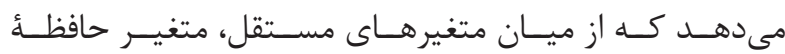

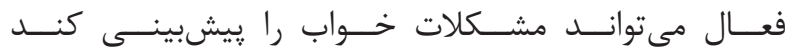
. $(P \leq \cdot / \cdot \Delta)$ 
جدول 9- نتايج ضريب همبستخى بِيرسون مشكلات خواب با حافظةٔ فعال و نكَهدارى توجه.

\begin{tabular}{|c|c|c|}
\hline معنى دارى & $\mathrm{r}$ & متغير \\
\hline$\cdot 1 \cdot \cdot$ & $-\cdot 19 \cdot r$ & كيفيت خواب با حافظة فعال \\
\hline$\cdot / T V Y$ & $\cdot 111 r$ & كيفيت خواب با نتههارى توجه \\
\hline
\end{tabular}

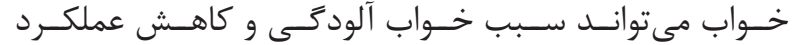

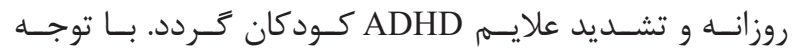

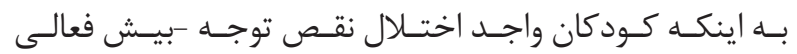

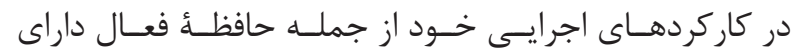

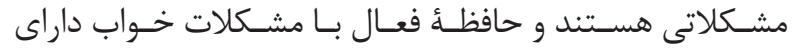

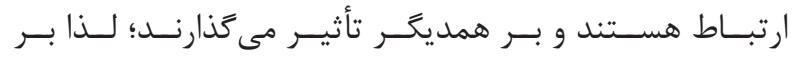

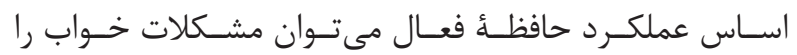

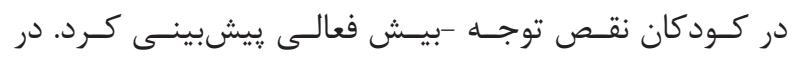

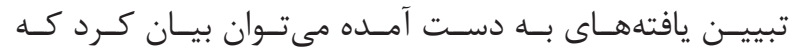

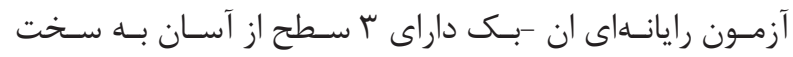

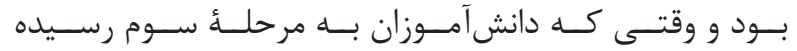

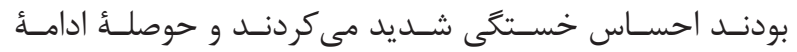

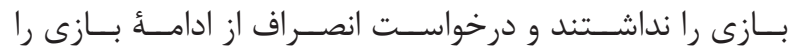

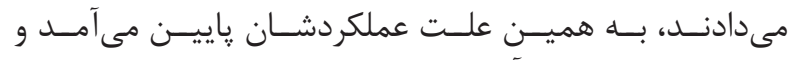

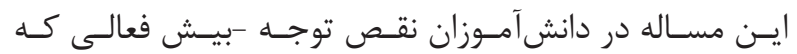

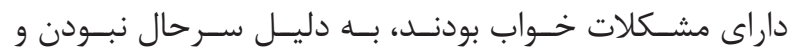

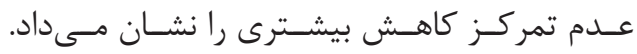

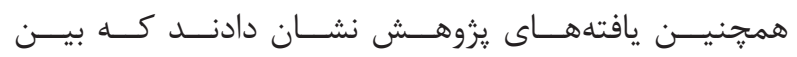

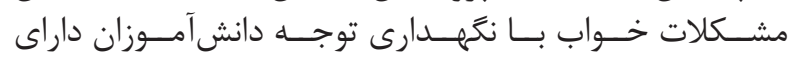

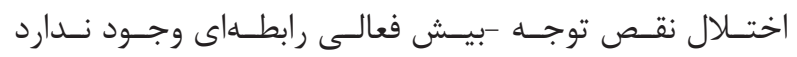

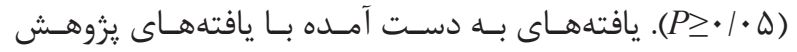

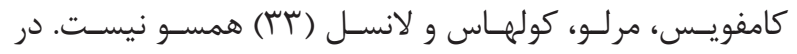

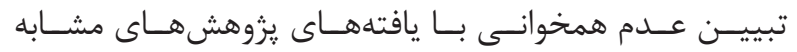

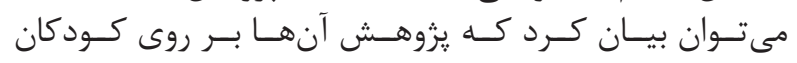

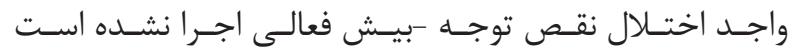

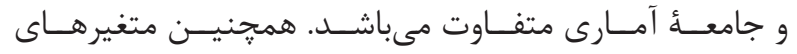

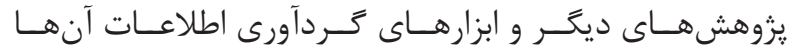

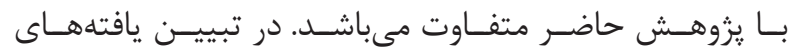

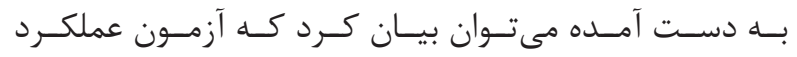

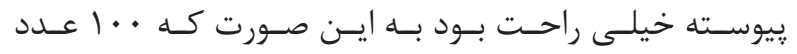

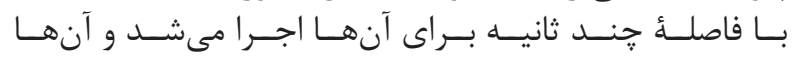

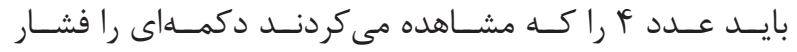

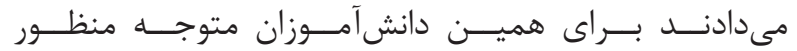

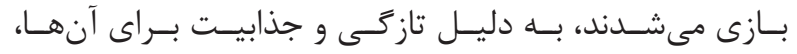

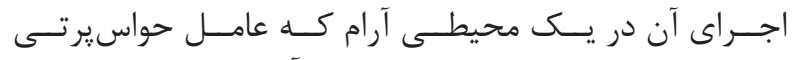

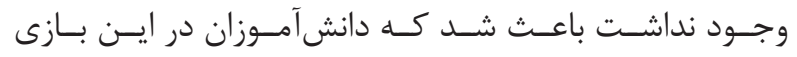

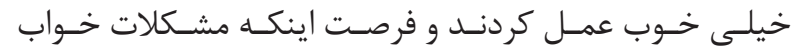

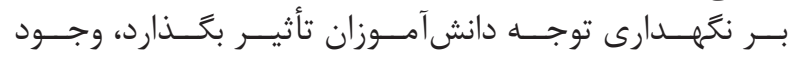

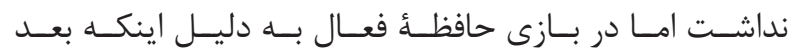

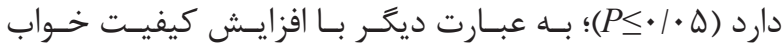

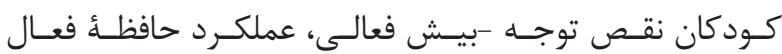

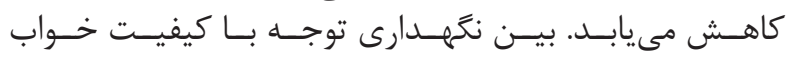

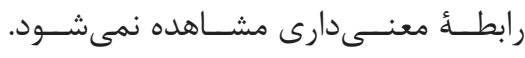
بحث و نتيجه كيرى

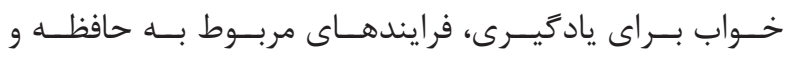

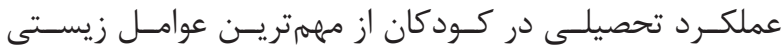

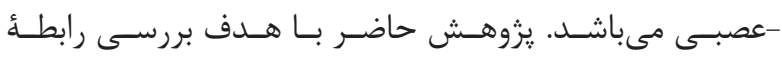

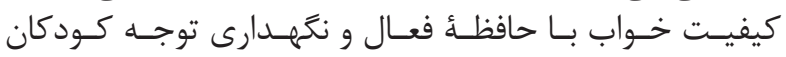

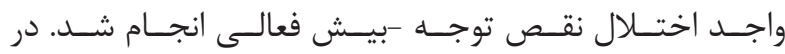

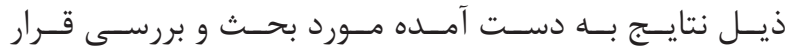

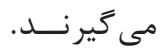

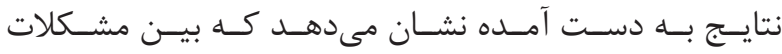

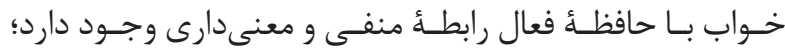

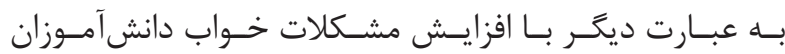

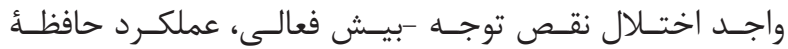

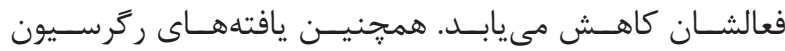

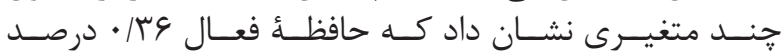

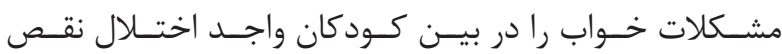

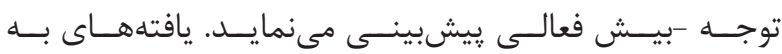

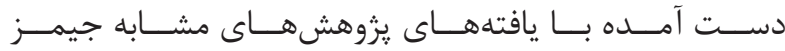

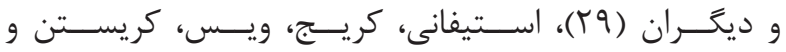

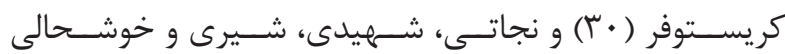

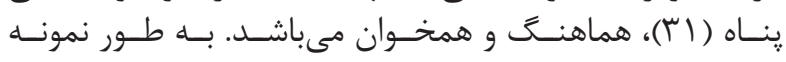

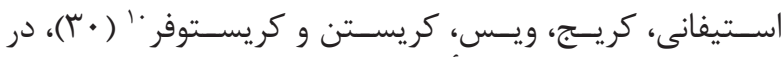

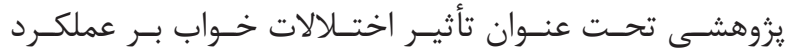

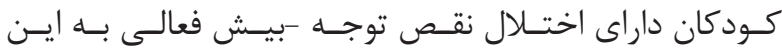

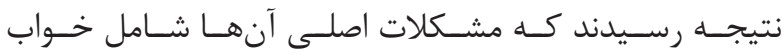

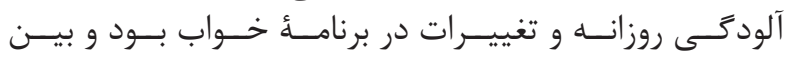

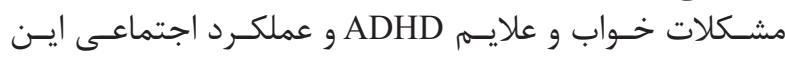

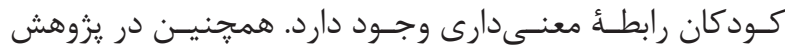

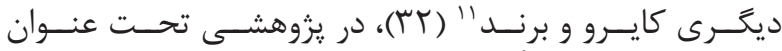

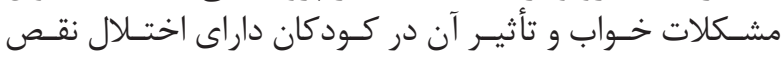

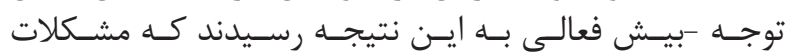

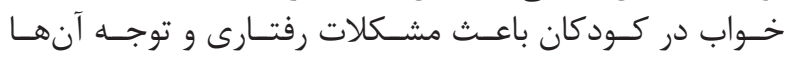

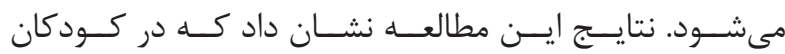

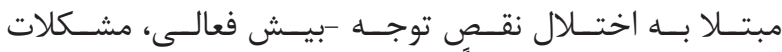

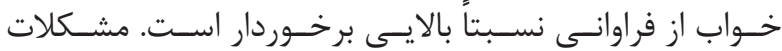

${ }^{10}$ Stephanie, Craig, Weiss, Kristen and Christopher

${ }^{11}$ Kirov and Brand 


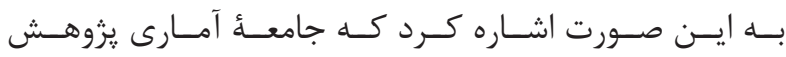

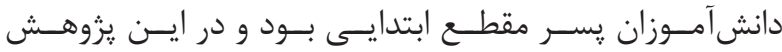

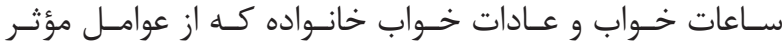

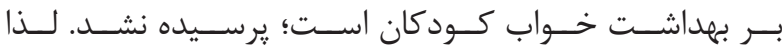

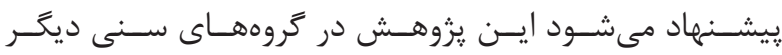

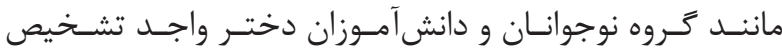

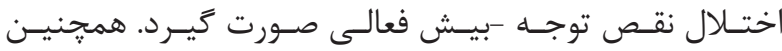

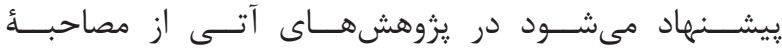

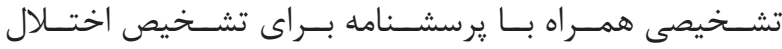

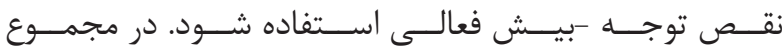

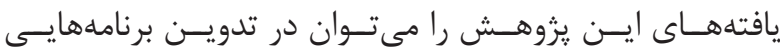

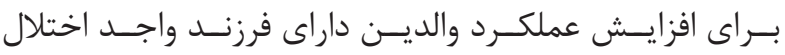

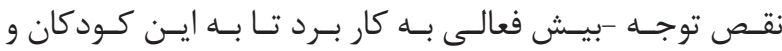

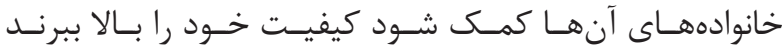

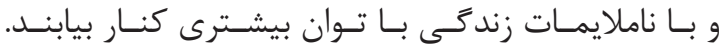

1. Tandon M, Pergjika D. Attention deficit hyperactivity disorder in preschool-age children. Child Adolesc Psychiatr Clin N Am. 2017; 26(3): 523-38.

2. American psychiatric association; diagnostic and statistical manual of mental disorders. $4^{\text {th }}$ ed. Washington, DC: Author. 2013.

3. Barkley RA, Murphy KV, Fischer M. ADHD in adult: what science says? New York: Guilford Press. 2008.

4. Nigg JT. What causes ADHD? understanding what goes wrong and why. New York: Guilford Press. 2006.

5. Carlson SM. Developmentally sensitive measures of executive function in preschool children. Dev Neuropsychol. 2005; 28(2): 595-616.

6. Alvarez JA, Emory E. Executive function and the frontal lobes: a meta-analytic review. Neuropsychol Rev. 2006; 16(1): 17-42.

7. Narimani M, Soleimani S. The effectiveness of cognitive rehabilitation on executive functions (working memory and attention) and academic achievement of students with math learning disorder. Journal of Learning Disabilities. 2013; 2(3): 115-91.

8. Hamzeloo M, Mashhadi A, Salehi Fadardi J. The prevalence of ADHD and comorbid disorders in Iranian adult male prison inmates. J Atten Disord. 2012; 20: doi: 10.1177/1087054712457991.

9. Kane MJ, Brown MW, Silva K. Controlled-attention view of working memory capacity: individual differences in memory span and the control of visual orienting. $J$

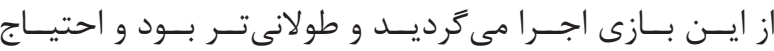

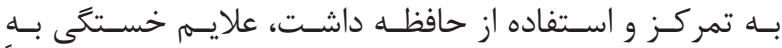

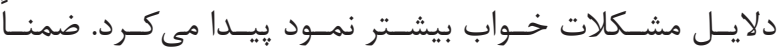

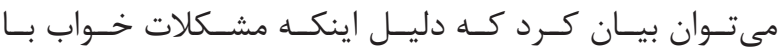

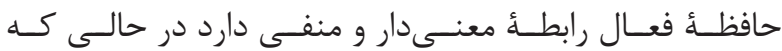

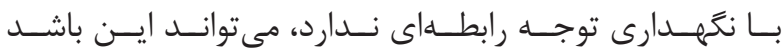

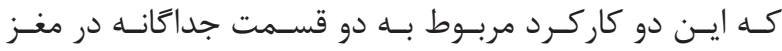

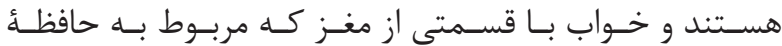

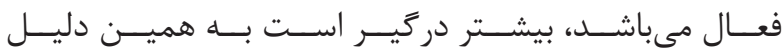

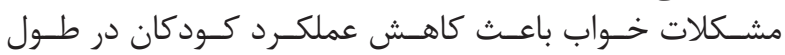

$$
\begin{aligned}
& \text { روز مىباشـــد. }
\end{aligned}
$$

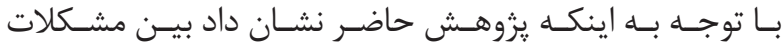

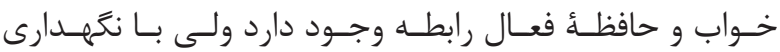

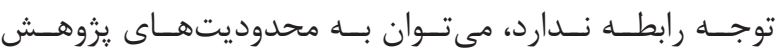

منابع

Exp Psychol Gen. 2017; 130(2): 169-83.

10. Baddeley A. "Working memory, thought, and action". NewYork: Oxford University Press. 2007.

11. Wells EL, Kofler MJ, Soto EF, Schaefer HS, Sarver DE. Assessing working memory in children with ADHD: Minor administration and scoring changes may improve digit span backward's construct validity. Res Dev Disabil. 2018; 72: 166-78.

12. Rapport MD, Kofler MJ, Alderson RM, Raiker JS. "Attention -deficit/ hyperactivity disorder". M Hersen, D Reitman. Handbook of Psychological Assessment, Case Conceptualization and Treatment. Children and Adolescents. Hoboken, NJ: Wiley. 2008; P. 349-404

13. Gathercole SE, Alloway TP. "Working memory and learning: A practical guide for teachers". NewYork: Sage. 2008.

14. Rapport MD, Bolden J, Kofler MJ, Sarver DE, Raiker JS, Alderson MR. Hyperactivity in boys with attentiondeficit/hyperactivity disorder (ADHD): a ubiquitous core symptom or manifestation of working memory deficits? J Abnorm Child Psychol. 2009; 37(4): 521-34.

15. Seidman LJ. Neuropsychological functioning archive of SID in people with ADHD across the lifespan. Clin Psychol Rev. 2006; 26(4): 466-85.

16. Alipor A, Baradaran M, Imanifar HR. The comparison of children with hyperactivity/ attention deficit, learning disabilities and normal children based on Children Wechsler IQ test components (new edition). Journal of Learning Disabilities. 2014; 4(3): 74-89. 
17. Kasaeian K, Kiamanesh A, Bahrami E. Comparing the performance of active memory and maintaining the attention of students with and without learning disabilities. Journal of Learning Disabilities. 2013; 3(4): 112-45.

18. Karimi L, Zare H. The effect of music on the sus tainable attention of children with attention deficit hyperactivity disorder. Journal of Educational and Educational Research. 2013; 1(2): 33-45.

19. Faught G, Conners F, Himmelberger M. Auditory and visual sustained attention in down syndrome. Res Dev Disabil. 2016; 53-54: 135-46.

20. Asadnia S, Sepehrianazar F, Mosarrezaii Aghdam A Saadatmand S. Comparison of sleep quality and migraine headaches in people with proper and improper and poor sleep. The Journal of Urmia University of Medical Sciences. 2013; 23(7): 799-806.

21. Cortese S, Konofal E, Yateman N, Mouren MC, Lecendreux M. Sleep and alertness in children with attention-deficit/hyperactivity disorder: a systematic review of the literature. Sleep. 2006; 29(4): 504-11.

22. Reynolds K, Patriquin M, Alfano C, Loveland K, Pearson D. Parent-reported problematic sleep behaviors in children with comorbid autism spectrum disorder and attention-deficit/hyperactivity disorder. Res Autism Spectr Disord. 2017; 39: 20-32.

23. Yoon S, Jain U, Shapiro C. Sleep in attention- deficit/ hyperactivity disorder in children and adults .past, present and future. Sleep Med Rev. 2012; 16(4): 371-88.

24. Haynes P, Christmann B, Griffith L. A single pair of neurons links sleep to memory consolidation in Drosophila melanogaster. Elife. 2015; 4. doi: 10.7554/ eLife. 03868.

25. Yazdi AS. The effectiveness of parenting education based on barclay's method on resuming and quality of life of mothers of children with acute disorders, attention deficit. Master's Thesis. Islamic Azad University of Khorasgan, Faculty of Humanities. 2017.

26. Derakhshanpour F, Vakili M, Nomoli, M, Hosseini, F. Sleep problems in children with hyperactivity disorder and attention deficit disorder. Journal of Gorgan University of Medical Sciences. 2014; 16: 52-9.

27. Nazifi M, Rasoulzadeh Tabatabaei K, Azadehfalah P, Moradi AS. Sustained attention and response inhibition in children with attention deficit/ hyperactivity disorder in comparison with normal children. Journal of Clinical Psychology. 2011; 2(10): 55-67.

28. Oraki M, Rahmanian M, Tehrani N, Heidari S. The effect of neurofeedback training on the improvement of active memory in children with acute disorders of attention deficit. Journal of Neuropsychiatry. 2015; 1: 41-52.

29. Waxmonsky JG, Mayes SD, Calhoun SL, FernandezMendoza J, Waschbusch DA, Bendixsen BH, et al. The association between disruptive mood dysregulation disorder symptoms and sleep problems in children with and without ADHD. Sleep Med. 2017; 37: 180-6.

30. Stephanie G, Craig Margaret D, Weiss Kristen L, Hudec Christopher G. The functional impact of sleep disorders in children with ADHD. Journal of Attention Disorders. 2017.

31 .Nejati V, Shahidi SH, Shiri E, Khoshhalipanah M. Comparing explicit and implicit memory in children with attention deficit- hyperactivity disorder and their typical developing counterparts. Journal of Research in Rehabilitation Sciences. 2014; 10(1): 131-8.

32. Kirov R, Brand S. Sleep problems and their effect in ADHD. Expert Rev Neurother. 2014; 14(3): 287-99.

33. Kamphuis J, Meerlo P, Koolhaas JM, Lancel M. Poor sleep as a potential causal factor in aggression and violence. Sleep Med. 2012; 13(4): 327-34. 